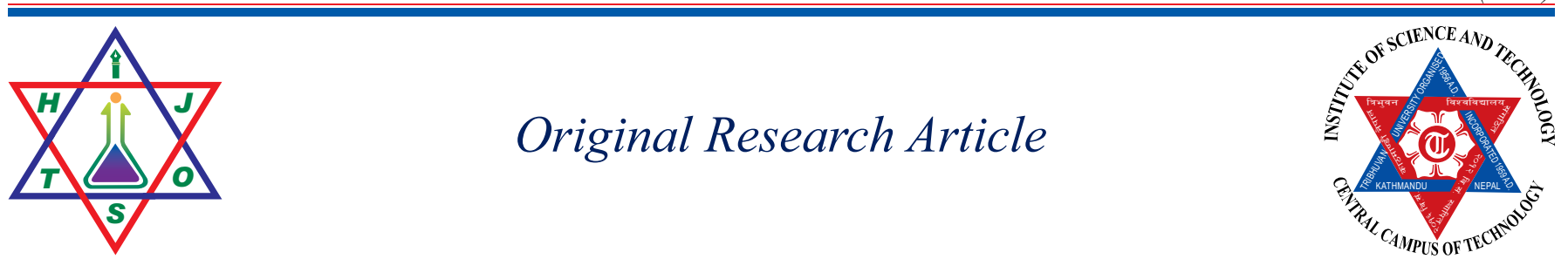

\title{
Freshwater Algae (Excluding Diatoms and Red Algae) from Hasina Wetland, Sundar \\ Haraicha, Morang, Nepal \\ Dilli Ram Rai ${ }^{l}$ and Shiva Kumar Rai ${ }^{*}$
}

${ }^{1}$ Phycology Research Lab, Department of Botany, Post Graduate Campus

Tribhuvan University, Biratnagar, Nepal

*Corresponding Author: Shiva Kumar Rai, Phycology Research Lab, Department of Botany, Post

Graduate Campus Biratnagar, Nepal,E-mail: sk.khaling@gmail.com

\section{Abstract:}

This paper described 44 freshwater algae (except diatoms and red algae) belonging to classes' cyanophyceae, chlorophyceae, charophyceae and euglenophyceae from Hasina wetland and its surrounding area, SundarHaraicha, Morang, Nepal. Samples were collected from Budhi Khola, Khayer Khola and ponds and marshy land in Hasina wetland. In total, the largest family was chlorophyceae represented by 30 taxa and the largest genus was Cosmarium represented by 6 taxa. The study showed that this area is rich in algal diversity.

Key words: Budhi Khola, Cyanophyceae, Khayer Khola, Spirulina, Trachelomonas.

\section{Introduction}

Algae are chlorophyllous thallophytes without true root, stem and leaves or leaf-like organs and commonly known as 'ponds scums', 'water mosses', 'frog spittle' or 'sea weeds'. They are unicellular to multicellular, without vascular tissue and have sex organ without jacket layer.

Algae are common in aquatic habitat but can also grow in many diverse habitats such as damp soil, moist wall, moist wood, tree trunk, and even under or inside porous rocks such as sand-stone and limestone. The occurrence of epiphytic, perforating algae of molluscs and calcarious rocks, snow, thermal water, epizoic condition and in symbiotic association had been reported. Specially, cyanophytes tolerate a wide range of temperatures and can be found growing on hot springs and snow bands or deep within polar-ice.

Algae vary greatly in size, ranging from $0.5 \mu \mathrm{m}$ unicellular to huge seaweeds (Macrocystis pyrifera) which may be of 100 feet or more. They are single celled, colonial, filamentous or frond like and bushy plant displaying endless degree of complexity. Algae also form mutually beneficial partnership with other organisms as with fungi to form lichens, with higher plants (Azolla leaf, coralloid root of Cycas), with coral reef (Zooxanthellae), etc. Algae provide oxygen and complex nutrients to their partner, and in return, they receive protection and simple nutrients.

Algae constitute an economically important group of plants. Their economic importance is many folds, both positive and negative. Spirulina sp. are protein rich blue green algae and are considered as future health food for world and now being sold in the market at a high price as a protein capsule (Rai, 2011). Nitrogen fixing capacity of algae can be utilized in agriculture as bio-fertilizer. About $25 \%$ of the total blue-green algae recorded from Nepal are found in paddy fields. They increase nitrogen level in paddy field resulting in higher productivity of the soil. It has been estimated that blue-green algae can add about 40 $\mathrm{kg}$ bound nitrogen to the soil/hectare/year (Van Den Hoek et al, 2009). Algae have been used for centuries, especially in Asian countries, for their purported powers to cure or prevent illness such as cough, gout, gallstones, goiter, hypertension and diarrhoea. Recently, algae have been surveyed for anti-cancer compounds. Diatoms also have been used in forensic medicine as their presence in the lungs can indicate a person's death due to drowning. Algae can also serve as bio-indicators of environmental problem in aquatic ecosystems. Water quality of many streams and rivers of central and eastern Nepal have been studied on the basis of diatom (Juttner et al, 2003).

Harmful activities of algae are formation of algal blooms 
which may deplete oxygen concentration in water as well as prevent light penetration for algae at lower depths preventing photosynthesis. Blue green algae grow in water tanks forming bottom gelatinous layers and hence creating problem in water supply.

Realizing various beneficial activities of algae, numerous taxonomic studies on algae have been carried out in the globe. In Nepal, the first major reports were published from Hirano (1963, 1969, and 1984) who reported 271 taxa (including 79 species of desmids) from Central and Eastern Nepal. Jha and Kargupta (2006) had described bluegreen algae from Sapta Koshi Basin but Hasina Wetland region has not been explored yet. Rai (2011a) studied algal flora of Betana wetland, Morang and reported 23 taxa including 6 diatoms which were new for the country. Similarly, Shrestha et al. (2013) had studied algae of Itahari and its adjoining area and reported 52 taxa. Rai $(2013,2014)$ also studied the algal flora including desmids of Koshi Tappu Wildlife Reserve. Hasina Wetland is favourable locality for freshwater red algae. Recently, Necchi et al, (2016) studied the phylogeny and morphology of Nemalionopsis shawii collected from the Hasina wetland. But, the other groups of algae from this locality have not been studied so far. Thus, the present work is aimed to explore the algal flora (excluding diatoms and red algae) of Hasina Wetland, Morang.

\section{Materials and Methods}

Algal samples (total 70) were collected by random sampling technique from five different localities, three from Hasina wetland and one each from Khayer Khola and Budhi Khola (Map 1) using plankton net (mesh size $0.5 \mathrm{~mm}$ ) and by squeezing submerged leaves and roots of aquatic macrophytes during the period of April 2015 to March 2016 (Baishakh to Chaitra, 2072 BS). They were labeled properly with sample number, date of collection, method of collection, locality etc. The latitude, longitude and altitude were measured with the help of GPS Garmin etrex. Necessary photographs of the collection sites were taken with the help of Canon Powershoot Camera. Peripheral aquatic vegetation as well as land vegetation were also noted. Water temperature and $\mathrm{pH}$ of each locality were also measured with the help of Mercury thermometer and portable Henna $\mathrm{pH}$ meter, respectively. Field note was maintained properly. The materials were brought to the Phycology Research Lab, P.G. Campus, Biratnagar and examined as soon as possible in living condition. The materials were then preserved on FAA solution as well as $4 \%$ formaldehyde solution for further detail studies. The slides of each sample were mounted in the glycerin jelly (Sharma, 1992). The prepared slides were examined under compound microscope in 100X, 400X and 1000X magnification. Green and blue green algae were stained with $1 \%$ methylene blue and iodine solution, respectively. Microphotographs were taken with the help of Olympus Ch20i microscope and Canon Digital Power Shot Camera using flash-light off and macro-mode under long exposure period.

Algal taxa were identified consulting various literatures and monographs such as Prescott (1951), Tiffany and Britton (1952), Desikachary (1959), Scott and Prescott (1961), Philipose (1967), Croasdale and Flint (1986,
1988), Croasdale et al, (1994), Prasad and Misra (1992) etc. Identification and recent updates of nomenclature were also approved by consulting online algae databases like http://algaebase.org, http://desmids.science4all, http://digicote.info etc. All the collected materials have been deposited in the Phycology Research Lab, Department of Botany, P.G. Campus, Biratnagar.

\section{Study Area}

Hasina wetland lies in the foothills of Churia range in the tropical zone about 4-5 km north from Bansbari Chowk (East-west highway) in Sundar Haraicha Municipality 7, Morang. This wetland is situated between Budhi Khola and Khayer Khola, to the south of Charkhose Jhadi covering an area of about 48 ha including the forest (Map 1). It is a natural wetland consisting of swamp, marsh, pond and streams. Hasina Wetland is almost rectangular in shape with its wide width toward north and slightly narrow width towards the south side. The water body coverage area is $3.3 \mathrm{ha}$.. The wetland type is lacustrine (lake/pond), palustrine (marsh/swamp) and riverine. The water flowing through the wetland has been used by making two dams, one within the wetland area (locality 5 ) and the other outside the wetland towards the south. The wetland is being used for irrigation for about 3200 ha land.

The first locality was Khayer Khola, a small stream in Gurung Tole, which lies to the east side from Hasina Wetland (Table 1). The second was Budhi Khola (Lati Khola) situated to the northwest side from Hasina Wetland. The third to fifth localities were the ponds and marshy places inside the Hasina Wetland. The third one was an old pond situated in the western side of wetland. 
The locality near the dinosaur statue was marked as situated in the eastern side which was the major source of locality no. 4 which consisted of flowing water with irrigation and also used for boating. But now this pond has shallow depth. The fifth locality was the largest pond been destroyed by the heavy flood occurred in 2017 .

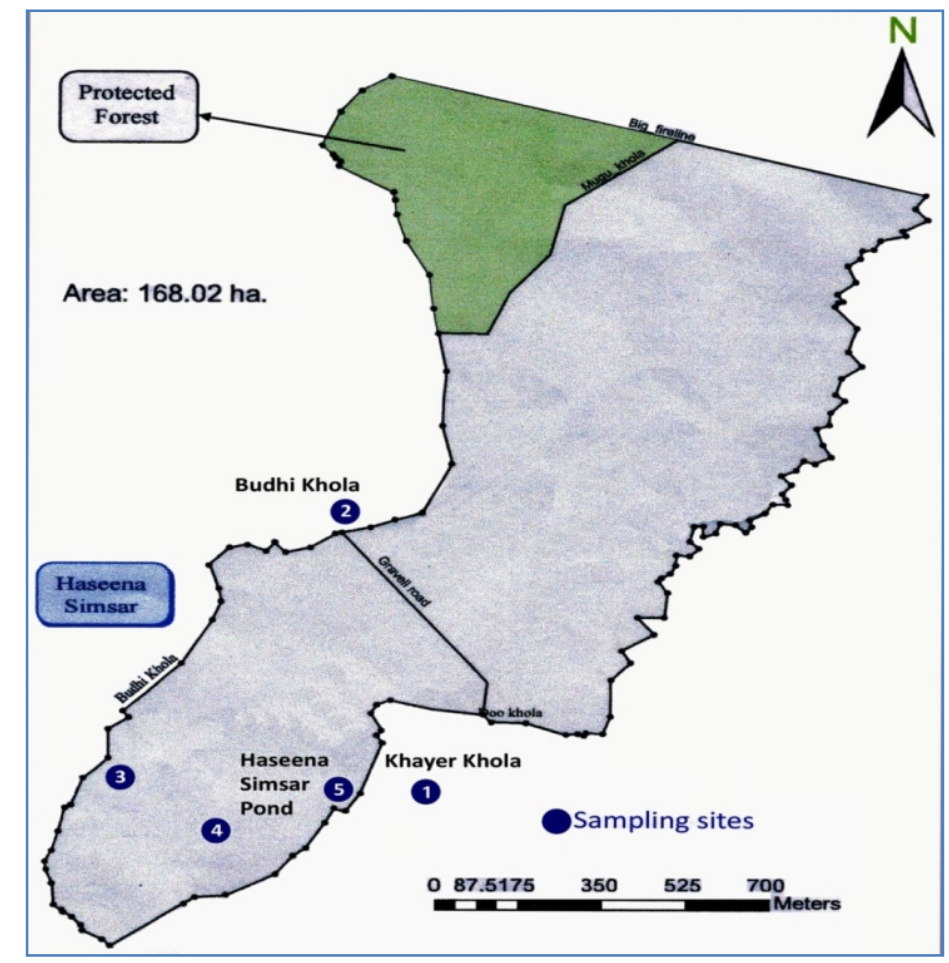

Map 1. Hasina Wetland and surrounding area showing sampling sites.

Table 1. Algae sampling sites with geographical position and water parameters.

\begin{tabular}{|c|c|c|c|c|c|c|c|c|}
\hline \multirow{2}{*}{$\begin{array}{l}\text { Locality } \\
\text { No. }\end{array}$} & \multirow[t]{2}{*}{ Name of locality } & \multirow[t]{2}{*}{ Habitat } & \multirow{2}{*}{$\begin{array}{l}\text { Geographical } \\
\text { position }\end{array}$} & \multirow{2}{*}{$\begin{array}{l}\text { Altitude } \\
(\mathrm{m})\end{array}$} & \multicolumn{2}{|c|}{ Water temp. $\left({ }^{\circ} \mathrm{C}\right)$} & \multicolumn{2}{|c|}{ Water $\mathrm{pH}$} \\
\hline & & & & & rainy & winter & rainy & winter \\
\hline 1 & Khayera Khola & Stream & $\begin{array}{l}26^{\circ} 41^{\prime \prime} 22.6^{\prime} \mathrm{N}, \\
87^{\circ} 19^{\prime \prime} 22.2^{\prime} \mathrm{E}\end{array}$ & 126 & 26 & 21 & 7.0 & 7.0 \\
\hline 2 & Budhi Khola & River & $\begin{array}{l}26^{\circ} 42^{\prime \prime} 05.4^{\prime} \mathrm{N}, \\
087^{\circ} 19^{\prime \prime} 06.3^{\prime} \mathrm{E}\end{array}$ & 132 & 28 & 22 & 6.5 & 7.0 \\
\hline 3 & Hasina Wetland & Pond & $\begin{array}{l}26^{\circ} 41^{\prime \prime} 33^{\prime} \mathrm{N}, \\
87^{\circ} 18^{\prime \prime} 48.7^{\prime} \mathrm{E}\end{array}$ & 118 & 32 & 15 & 6.5 & 6.5 \\
\hline 4 & Hasina Wetland & Stream & $\begin{array}{l}26^{\circ} 41^{\prime \prime} 34^{\prime} \mathrm{N} \\
87^{\circ} 19^{\prime \prime} 04.7^{\prime} \mathrm{E}\end{array}$ & 119 & 28 & 22 & 7.0 & 7.0 \\
\hline 5 & Hasina Wetland & Pond & $\begin{array}{c}26^{\circ} 41^{\prime \prime} 35.8^{\prime} \mathrm{N} \\
87^{\circ} 19^{\prime \prime} 07.5^{\prime} \mathrm{E}\end{array}$ & 122 & 27 & 17 & 6.5 & 7.0 \\
\hline
\end{tabular}

The climate of this area is tropical or subtropical mucronata, Alternanthera philoxeroides, etc. Invasive monsoonal type and experiences three distinct seasons i.e., alien species such as Banmara Chromolaena odorata, summer (February to May), rainy (June to September) and Agaratum conyzoides, Mikenia mikrantha and Lantana winter (October to January). The area has maximum camara are growing dominantly along the marginal area of temperature in May and minimum in January. Similarly, it the wetland (Siwakoti et al, 2012). The major land experiences maximum rainfall in July and minimum in vegetation is composed of tropical plants viz., Shorea December. robusta, Syzygium cumini, Anthocephalus chinensis, Diospyros species, Cassia fistula, Schleichera oleosa, This wetland provides habitat to different species of Dalbergia sissoo, Acacia catechu, Bombax ceiba, Aegle floating and submerged macro-flora. Common floating marmelos, Trewia nudifloria, Callicarpa arborea, spices are Eichhornia crassipes, Pistia stratoites, Mallotus phillipensis, etc. This wetland also provides a Monochoria sp, Lemna minor, Vallisnaria natans, etc. good habitat for many rare flora and fauna.

Emergant species in marshy area are Schoenopletus 


\section{Results and Discussion}

Algal flora (excluding diatoms) of Hasina Wetland and its periphery area have been studied. A total of 44 freshwater algae under 28 genera belonging to class cyanophyceae,

chlorophyceae, charophyceae and euglenophyceae were reported from 5 different localities (Table 2).

Table 2. Classification (Guiry and Guiry, 2018) of algae reported from Hasina Wetland.

\begin{tabular}{|c|c|c|c|c|c|}
\hline \multicolumn{2}{|c|}{ Divisn Class } & Order & Family & Genera & Species \\
\hline \multirow{11}{*}{$\begin{array}{l}\frac{\pi}{2} \\
\text { ct } \\
\frac{0}{0} \\
\frac{0}{\pi} \\
\frac{\pi}{0}\end{array}$} & \multirow{11}{*}{ 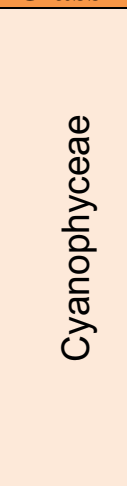 } & \multirow{11}{*}{$\begin{array}{l}\frac{0}{0} \\
\frac{\pi}{\pi} \\
\frac{0}{0} \\
0 \\
\frac{0}{2}\end{array}$} & eShroococcacea & \multicolumn{2}{|c|}{ e Chroococcu\$. C. turgidus } \\
\hline & & & \multirow{8}{*}{ 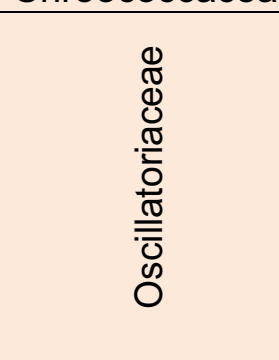 } & Spirulina & 2. S. subsalsa \\
\hline & & & & Oscillatoria & 3. O. limosa \\
\hline & & & & & 4. O. princeps \\
\hline & & & & & 5. O. sancta \\
\hline & & & & & 6. O. subbrevis \\
\hline & & & & Phormidium & 7. P. autumnale \\
\hline & & & & Lyngbya & 8. L. birgei \\
\hline & & & & Nostoc & 9. N. microscopicum \\
\hline & & & Rivulariaceae & Gloeotrichia & 10. G. echinulata \\
\hline & & & Stigonematacea & a8tigonema & 11. S. ocellatum \\
\hline \multirow{30}{*}{$\begin{array}{l}\frac{\pi}{2} \\
\frac{1}{0} \\
\frac{0}{0} \\
\frac{0}{\frac{0}{0}} \\
U\end{array}$} & \multirow{29}{*}{ 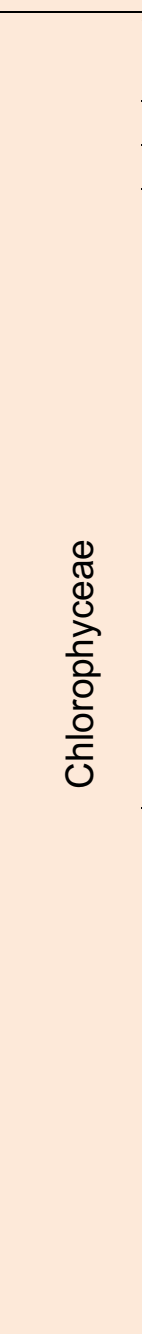 } & Volvocales & Volvocaceae & Eudorina & 12. E. elegans \\
\hline & & & & Pandorina & 13. P. morum \\
\hline & & Tetrasporale & sPalmellaceae & Sphaerocysti & s14. S. schroeteri \\
\hline & & Oedogoniale & Sedogoniaceae & Oedogonium & 15. O. sp. \\
\hline & & \multirow{13}{*}{ 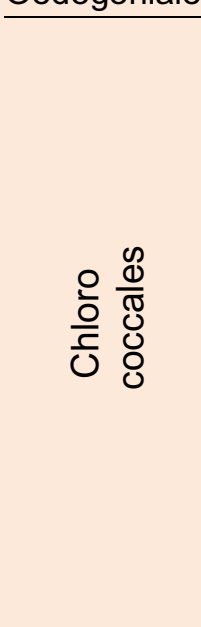 } & Hydrodictyacea & Pediastrum & 16. P. tetras v. tetraodon \\
\hline & & & Oocystaceae & Gloeotaeniun & 17. G. Ioitlesbergerianum \\
\hline & & & & Nephrocytiun & 18. N. agardhianum \\
\hline & & & Dictyosphaeriace & destella & 19. W. botryoides \\
\hline & & & Selenastraceae & Ankistrodesmı & 1s0. A. spiralis \\
\hline & & & & & 21. A. spiralis $v$. fasciculatus \\
\hline & & & Coelastraceae & Coelastrum & $\begin{array}{l}\text { 22. C. cambricum } \\
\text { 23. C. cambricum v. intermediur }\end{array}$ \\
\hline & & & Scenedesmace & aerucigenia & 24. C. tetrapedia \\
\hline & & & & Scenedesmu & s25. S. acutiformis \\
\hline & & & & & 26. S. arcuatus \\
\hline & & & & & 27. S. armatus v. boglariensis \\
\hline & & & & & 28. S. bijugatus \\
\hline & & & & & $\overline{29 .}$ S. bijugatus v. gravenitzii \\
\hline & & \multirow{12}{*}{ 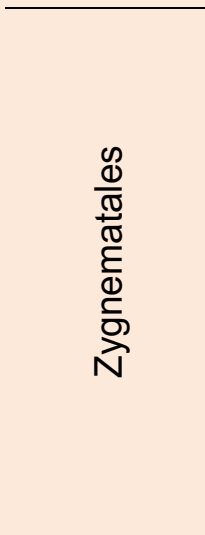 } & Desmidiaceae & Closterium & 30. C. dianae \\
\hline & & & & & 31. C. ehrenbergii \\
\hline & & & & & 32. C. praelongum v. brevius \\
\hline & & & & Pleurotaeniu & n33. P. trabecula \\
\hline & & & & Cosmarium & 34. C. blyttii \\
\hline & & & & & 35. C. impressulum \\
\hline & & & & & 36. C. javanicum \\
\hline & & & & & 37. C. Iundellii v. circulare \\
\hline & & & & & 38. C. Iundellii v. ellipticum \\
\hline & & & & & 39. C. sublateriundatum \\
\hline & & & & Staurastrum & 40. S. disputatum v. sinense \\
\hline & & & & Hyalotheca & 41. H. dissiliens \\
\hline & \multicolumn{2}{|c|}{$\begin{array}{l}\text { Charo Charales } \\
\text { phyceae }\end{array}$} & Characeae & Chara & 42. C. sp. \\
\hline \multirow{2}{*}{\multicolumn{3}{|c|}{$\begin{array}{l}\text { EuglendEuglendEuglenales } \\
\text { phyta phyceae }\end{array}$}} & Euglenaceae & Phacus & 43. P. pseudoswirenkoi \\
\hline & & & & Trachelomon & a\$4. T. armata v. longispina \\
\hline
\end{tabular}


The largest class was chlorophyceae represented by 30 taxa, followed by cyanophyceae (11 taxa), eulgenophyceae ( 2 taxa) and charophyceae with single taxa. In chlorophyceae, the desmid (Familly Desmidiaceae) algae were the dominant as usual. Among the genera, Cosmarium had the maximum number of taxa algae (6) followed by Scenedesmus (5), Oscillatoria (4), and Closterium (3). Genera Oedogonium and Chara could not be identified upto species level due to the lack of reproductive structures. Similarly, species of Spirogyra could not be confirmed due to the lack of literature. Taxonomic descriptions of all algae are as follow:

Chroococcus turgidus (Kuetz.) Nag. (Figs. 1-2) Desikachary 1959, P. 101, Pl.26, Fig.6.

Colony of 2-4 cells; cells $15-50 \mu \mathrm{m}$ long with sheath, 8$32 \mu \mathrm{m}$ in diameter without sheath.

Locality no: 2, Sample no: 4, Date of collection: 2072/04/12.

Spirulina subsalsa Oerst. ex Gom. (Fig. 3) Presscot 1951, P. 480, Pl, 108, Fig. 14.

Trichomes 1-2 $\mu \mathrm{m}$ in diameter, closely spiral; spiral 3-5 $\mu \mathrm{m}$ wide.

Locality no: 3, Sample no: 5, Date of collection: 2072/04/12.

Oscillatoria limosa (Roth) Agardh (Fig. 4) Desikachary 1959, P. 206, Pl. 42, Fig. 11.

Trichomes straight; cells $3.7-5 \mu \mathrm{m}$ long, $12-18 \mu \mathrm{m}$ in diameter, not constricted, apical cell rotund.

Locality no: 4, Sample no: 5, Date of collection: 2072/04/12.

Oscillatoria princeps Vaucher ex Gom. (Fig. 5) Tiffany and Britton 1952, P. 344, Pl. 93, Fig. 1080

Trichomes slightly taperig; cells 4-8.7 $\mu \mathrm{m}$ long, 32-55 $\mu \mathrm{m}$ in diameter, not constricted; apical cell not capitate.

Locality no: 5, Sample no: 4, Date of collection: 2072/04/12.

Oscillatoria sancta (Kutz.) Gom. (Fig. 6) Prescott 1951, P. 490, Pl. 110, Fig. 4

Trichomes straight; cells $4-5 \mu \mathrm{m}$ long, $11-13 \mu \mathrm{m}$ in diameter, slightly constricted; apical cell somewhat capitate with a calyptra and with a much thickened outer membrane.

Locality no: 1, Sample no: 7, Date of collection: 2072/09/05

Oscillatoria subbrevis Schm. (Fig. 7) Desikachary 1959, P. 207, Pls. 37 and 40, Fig. 2

Trichome straight, not tapering; cells 1-2 $\mu \mathrm{m}$ long, 5-6 $\mu \mathrm{m}$ in diameter, short, with frequent necridia; apical cell rounded, not capitates and without a calyptras.

Locality no: 2, Sample no: 5, Date of collection: 2072/04/12.

Phormidium autumnale (Ag.) Gomont [Microcoleus autumnalis (Gom.) Strun. et al.] (Fig. 8) Prescott 1951, P. 493, P1. 107, Fig. 19.

Filaments straight or curved, flexuous, slightly tapering; cell 2-5 $\mu \mathrm{m}$ long, 4-7 $\mu \mathrm{m}$ in diameter, not constricted, apex straight or curved, capitate, with a calyptras.

Locality no: 3, Sample no: 09, Date of collection: 2072/09/05

Lyngbya birgei Smith [Limnoraphis birgei (Smith) Kom. et al.] (Fig. 9) Tiffany and Britton 1952, P. 339, Pl. 92, Figs. 1066, 1067

Filaments straight, $24 \mu \mathrm{m}$ in diameter; sheaths thick, firm, not lamellated, extending beyond apices; trichomes 20-24 $\mu \mathrm{m}$ in diameter, not constricted; cells 3-7 $\mu \mathrm{m}$ long, short, disciform.

Locality no: 5, Sample no: 07, Date of collection: 2072/09/05.

Nostoc microscopicum Carm. ex Born. \& Flah. (Fig. 10) Prescott 1951, P. 523, Pl. 120, Fig. 3-5.

Colony minute, globose or ovoid; trichomes loose in colorless mucilage; cells globose, 5-8 $\mu \mathrm{m}$ in diameter, loosely arranged; heterocysts $7.3 \mu \mathrm{m}$ in diameter, subspherical or ovate.

Locality no: 4, Sample no: 03, Date of collection: 2072/04/12

Gloeotrichia echinulata (Smith) Rich. (Fig. 11) Desikachary 1959, P. 556, P1. 116, Figs. 9, 10. 
Thallus spherical, $0.5-7 \mathrm{~mm}$ diam.; filaments loose, radial; sheath not lamellated, colourless; trichome 8-10 $\mu \mathrm{m}$ broad at base, with a long hair; cells cylindrical with gas vacuoles.

Locality no: 3, Sample no: 08, Date of collection: 2072/09/05.

Stigonema ocellatum (Dillw.) Thuret ex Born. et Flah. (Fig. 12) Prescott 1951, P. 548, Pl. 130, Figs. 5, 6.

Filaments 35-45 $\mu \mathrm{m}$ wide, brown tufts, with long curved brances; trichomes uniseriate; heterocyst lateral; cells 20$30 \mu \mathrm{m}$ broad, quadrate-globoses to globose.

Locality no: 3, Sample no: 08, Date of collection: 2072/09/05.

Eudorina elegans Ehr. (Fig. 13) Tiffany and Britton 1952, P. 17, P1. 2, Fig. 14

Colony spherical, up to $200 \mu \mathrm{m}$ diam., $16-32$ celled; cells ovoid, $10-20 \mu \mathrm{m}$ broad, evenly disposed or arranged in transverse series, usually lying near the periphery of the envelope.

Locality no: 4, Sample no: 08, Date of collection: 2072/09/05.

Pandorina morum (Mull.) Bory (Fig. 14) Prsecott 1951, P. 75, P1. 1, Fig. 23

Colony motile, sub-spherical to ellipsoid, about $220 \mu \mathrm{m}$ diam., 16 celled; cells pyriform, 12-17 $\mu \mathrm{m}$ long, 10-15 $\mu \mathrm{m}$ broad, mutually compressed and enclosed in a gelatinous envelope.

Locality no: 5, Sample no: 05, Date of collection: 2072/04/12.

Sphaerocystis schroeteri Chodat (Fig. 15) Prescott 1951, P. 83, P1. 3, Figs. 6, 7

Colony spherical, up to $500 \mu \mathrm{m}$ diam., 4-8 celled, arranged periphery in hyaline envelop; cells spherical, 6$20 \mu \mathrm{m}$ in diameter.

Locality no: 5, Sample no: 05 , Date of collection: 2072/04/12.

Oedogonium sp. (Fig. 16) Prasad and Misra 1992, P. 66.

Filament unbranched, attached; cells cylindrical or enlarged toward the anterior end with one or more ring like scars; chloroplast a parietal reticulum with many pyrenoids.

Locality no: 4, Sample no: 04, Date of collection: 2072/04/12.

Pediastrum tetras var. tetraodon (Corda) Hansg. (Fig. 17) Philipose 1967, P. 129, Fig. 45 d, e, g.

Colony circular, 4-8-16 celled, 8 celled colony $20-33 \mu \mathrm{m}$ in diam.; cells $5-15 \mu \mathrm{m}$ broad, no intercellular spaces; marginal cells 2 lobes, deep incision; inner cells 4-6 sided, 1 incision.

Locality no: 1, Sample no: 06, Date of collection: 2072/09/05

Gloeotaenium loitlesbergerianum Hansg. (Figs. 18-19) Komarek 1983, P. 116, P1. 17, Fig 36.

Colony spherical or quadrangular ovate, 2-8 celled, 8 celled colony $80 \mu \mathrm{m}$ long, $70 \mu \mathrm{m}$ in diam.; cells globose or ellipsoid, 18-25 $\mu \mathrm{m}$ in diam., compactly and cruciately arranged.

Locality no: 3, Sample no: 03, Date of collection: 2072/04/12.

Nephrocytium agardhianum Nag. (Fig. 20) Philipose 1967, P. 189, Fig. 104

Colony ovate, $2-8$ celled; cells $8-18 \mu \mathrm{m}$ long, $2-7 \mu \mathrm{m}$ in diam., cylindrical or reniform, twisting so as to give a spiral arrangement in an old mother cell wall.

Locality no: 4, Sample no: 04, Date of collection: 2072/09/05

Westella botryoides (West) de Wild. (Fig. 21) Prescott 1951, P. 237, Pl. 53, Fig. 14.

Colony 40-80 celled; cells spherical, 3-9 $\mu \mathrm{m}$ in diam., quadrately arranged in groups of 4 , the groups loosely connected by persistent remains of old mother cell walls.

Locality no: 1, Sample no: 06, Date of collection: 2072/09/05.

Ankistrodesmus spiralis (Trun.) Lemm. (Fig. 22) Philipose 1967, P. 210, Fig. 119 a.

Cells 25-35 $\mu \mathrm{m}$ long, 2-3 $\mu \mathrm{m}$ broad, spindle, spirally twisted into bundles of $4-16$ cells. 
Locality no: 1, Sample no: 06, Date of collection: 2072/09/05.

Ankistrodesmus spiralis (Turn.) Lemm. var. fasciculatus Smith (Figs. 23-24) Prasad and Misra 1992, P. 27, Pl. 4, Fig. 8.

Colony $75-180 \mu \mathrm{m}$ in diam., cells $55-110 \mu \mathrm{m}$ long, 3-4.5 $\mu \mathrm{m}$ broad, curved or sigmoid, middle part twisted around each other in colonies of $34-50$ cells, apical portion of cell free.

Locality no: 2, Sample no: 07, Date of collection: 2072/09/05.

Coelastrum cambricum Arch. (Fig. 25) Prescott 1951, P. 229, Pl. 53, Fig. 2.

Colony spherical, 8-128 celled; each cells $10-20 \mu \mathrm{m}$ in diam., joined to other cells by 6 projections of sheath, triangular intercellular space; outer free wall with truncate projection.

Locality no: 4, Sample no: 04, Date of collection: 2072/04/12.

Coelastrum cambricum var. intermedium (Boh.) West (Fig. 26) Prasad and Misra 1992, P. 30, P1. 4, Fig. 5.

Colony spherical, $60-66 \mu \mathrm{m}$ in diam., 32 celled; cells $14-$ $21 \mu \mathrm{m}$ in diam., spherical in middle, subspherical at periphery with slightly thick, blunt and rounded projections.

Locality no: 5, Sample no: 10, Date of collection: 2072/09/05.

Crucigenia tetrapedia (Kirch.) West (Fig. 27) Philipose 1967, P. 240, Fig. 151.

Colony flat, 4 triangular cells cruciately arranged leaving a central space, frequently form plate of 16 cells; cells 4.5-9 $\mu \mathrm{m}$ in diam., outer walls straight, and angles acutely rounded.

Locality no: 4, Sample no: 08, Date of collection: 2072/09/05.

Scenedesmus acutiformis Schroed. [Acutodesmus acutiformis (Schroed.) Tsarenko et John] (Fig. 28) Tiffany and Britton 1952, P. 123, P1. 35, Fig. 356

Colony flat; cells fusiform-elliptic, cylindrical, 17-22.5 $\mu \mathrm{m}$ long, 6-8 $\mu \mathrm{m}$ broad, arranged in a single series of 2-8, poles sharply pointed.

Locality no: 4, Sample no: 04, Date of collection: 2072/04/12.

Scenedesmus arcuatus (Lemm.) Lemm. (Fig. 29) Prescott 1951, P. 275, P1. 62, Fig. 8.

Cells oblong ovate, 10-15 $\mu \mathrm{m}$ long, 4-8 $\mu \mathrm{m}$ broad, arranged forming a curve of 4-16 cells, lateral walls contact along $1 / 3$ to $1 / 2$ of length; poles boradly rounded.

Locality no: 2, Sample no: 04, Date of collection: 2072/04/12.

Scenedesmus armatus var. boglariensis Horto. (Fig. 30) Philipose 1967, P. 264, Figs. 171 g, j.

Colony flat, slightly curved, 2-4 celled in a linear series; cells 8-14 $\mu \mathrm{m}$ long, 2.4-4.2 $\mu \mathrm{m}$ broad with longitudinal rib; terminal cells with a spine, one straight, other slightly curved.

Locality no: 3, Sample no: 08, Date of collection: 2072/09/05

Scenedesmus bijugatus (Turp.) Kutz. (Fig. 31) Prasad and Misra 1992, P.35, P1.5, Fig.1

Colony flat, slightly curved, 2-4-8 celled, in a linear series; cells oblong-ellipsoid to ovoid, cells 7-23 $\mu \mathrm{m}$ long, 3.5-7 $\mu \mathrm{m}$ broad, ends broadly rounded.

Locality no: 4, Sample no: 04, Date of collection: 2072/04/12.

Scenedesmus bijugatus var. gravenitzii (Barn.) Chodat (Fig. 32) Philipose 1967, P. 254, Figs. 164 a, b.

Colony 4-8 celled, in alternate series, adjacent cells contact along a short portion of their length; cells fusiform, ellipsoid, ovoid, 10-16.7 $\mu \mathrm{m}$ long, 4.5-7.9 $\mu \mathrm{m}$ broad, obtuse poles.

Locality no: 5, Sample no: 05, Date of collection: 2072/04/12.

Closterium dianae Ehr. ex Ralfs (Fig. 33) Tiffany and Britton 1952, P. 169, Pl. 52, Fig. 548

Cells 105-303 $\mu \mathrm{m}$ long, $16-35 \mu \mathrm{m}$ broad, outer margins with $112-125^{\circ}$ arced, inner slightly tumid, gradually attenuated towards obtusely rounded apex. 
Locality no: 1, Sample no: 01, Date of collection: 2072/04/12.

Closterium ehrenbergii Menegh. ex Ralfs (Fig. 34) Scott and Prescott 1961, P. 11, Pl. 2, Fig. 2.

Cells large, stout, 450-530 $\mu \mathrm{m}$ long, $65-80 \mu \mathrm{m}$ broad, moderately curved, outer margins $92-110^{\circ}$ arced, inner margin inflated in middle, attenuate towards obtusely rounded apex.

Locality no: 1, Sample no: 01, Date of collection: 2072/04/12.

Closterium praelongum var. brevius (Nordst.) Krieg. (Fig. 35) Croasdale and Flint 1986, P. 67, P1. 9, Figs.4-7.

Cells long, 250-360 $\mu \mathrm{m}$ long, $17-23 \mu \mathrm{m}$ broad, slightly curved, straight at middle, gradually attenuated to recurved apical region, apices rounded-truncate; isthmus 3-3.5 $\mu$ m wide.

Locality no: 1, Sam ple no: 01, Date of collection: 2072/04/12.

Pleurotaenium trabecula (Ehr.) Nag. (Figs. 36-37) Kouwets 1987, P. 208, P1. 6, Fig. 7.

Cells straight, 430-510 $\mu \mathrm{m}$ long, 28-40 $\mu \mathrm{m}$ broad; semicells with slight basal inflation, gradually attenuated to rotudo-truncate apices, cell apices without tubercles.

Locality no: 5, Sample no: 10, Date of collection: 2072/09/05.

Cosmarium blyttii Wille (Fig. 38) Prasad and Misra 1992, P. 155, P1. 24, Figs. 2-3.

Cell small, 14-17.5 $\mu \mathrm{m}$ long, 13-14.5 $\mu \mathrm{m}$ broad, deeply constricted; semicell trapiziform-semicircular, basal angles subrectangular, sides with 4 crenates; apex truncate, 4 crenates.

Locality no: 5, Sample no: 10, Date of collection: 2072/09/05.

Cosmarium impressulum Elfv. (Fig. 39) Croasdale and Flint 1988, P. 71, Pl. 40, Figs. 16-19.

Cells small, $20.5 \mu \mathrm{m}$ long, $15 \mu \mathrm{m}$ broad, deeply constricted; sinus narrow, linear; semicells vsubquadrate, tri-undulate marigin, undulation sub-acute; apex retuse with rounded angles.
Locality no: 5, Sample no: 10, Date of collection: 2072/09/05

Cosmarium javanicum Nordst. (Fig. 40) Nurul Islam and Yusuf Haroon 1980, P. 576, Pl. 11, Figs. 156-157; Pl. 13, Fig. 179.

Cells large, 90-188 $\mu \mathrm{m}$ long, 45-89 $\mu \mathrm{m}$ broad, oblongelliptic, sides convex; sinus shallow, closed; semicells in side and end views elliptic; isthmus $35-52 \mu \mathrm{m}$ wide.

Locality no: 4, Sample no: 04, Date of collection: 2072/04/12.

Cosmarium lundellii var. circulare (Reins.) Krieg. (Fig. 41) Bharati and Hegde 1982, P. 744, Pl. 1, Fig. 2

Cells $46 \mu \mathrm{m}$ long, $37.8 \mu \mathrm{m}$ broad, circular in front view and elliptic in top view; cell-wall minutely punctate; isthmus $13.5 \mu \mathrm{m}$ wide.

Locality no: 4, Sample no: 04, Date of collection: 2072/04/12.

Cosmarium lundellii var. ellipticum West et West (Figs. 42-43) Scott and Prescott 1961, P. 61, P1. 25, Fig. 8

Cells $57-73 \mu \mathrm{m}$ long, 40-58 $\mu \mathrm{m}$ broad, more or less broadly ellipsoid in front view, elliptical in top view; cell apex is somewhat flat; isthmus $15-23 \mu \mathrm{m}$ wide.

Locality no: 2, Sample no: 02 , Date of collection: 2072/04/12.

Cosmarium sublateriundatum West et West (Fig. 44) Bando et al. 1989, P. 21, Fig. 7 e.

Cells 41-46 $\mu \mathrm{m}$ long, 36-40 $\mu \mathrm{m}$ broad, deeply constricted; semicells subtrapeziform; lateral margins 67 undulations; apex truncate, 2 undulations; isthmus 12.5-13.5 $\mu$ m wide.

Locality no: 4, Sample no: 08 , Date of collection: 2072/09/05

Staurastrum disputatum var. sinense (Lutk.) West et West (Figs. 45-46) Croasdale et al. 1994, P. 95, Pl. 81, Figs. 1014.

Cells small, 17-24 $\mu \mathrm{m}$ long, 19-25 $\mu \mathrm{m}$ broad; sinus widely open, minutely notched interior; semicells cuneiform; upper part horizontal, bluntly cylindrical; isthmus 5.5-9 $\mu \mathrm{m}$ wide. 

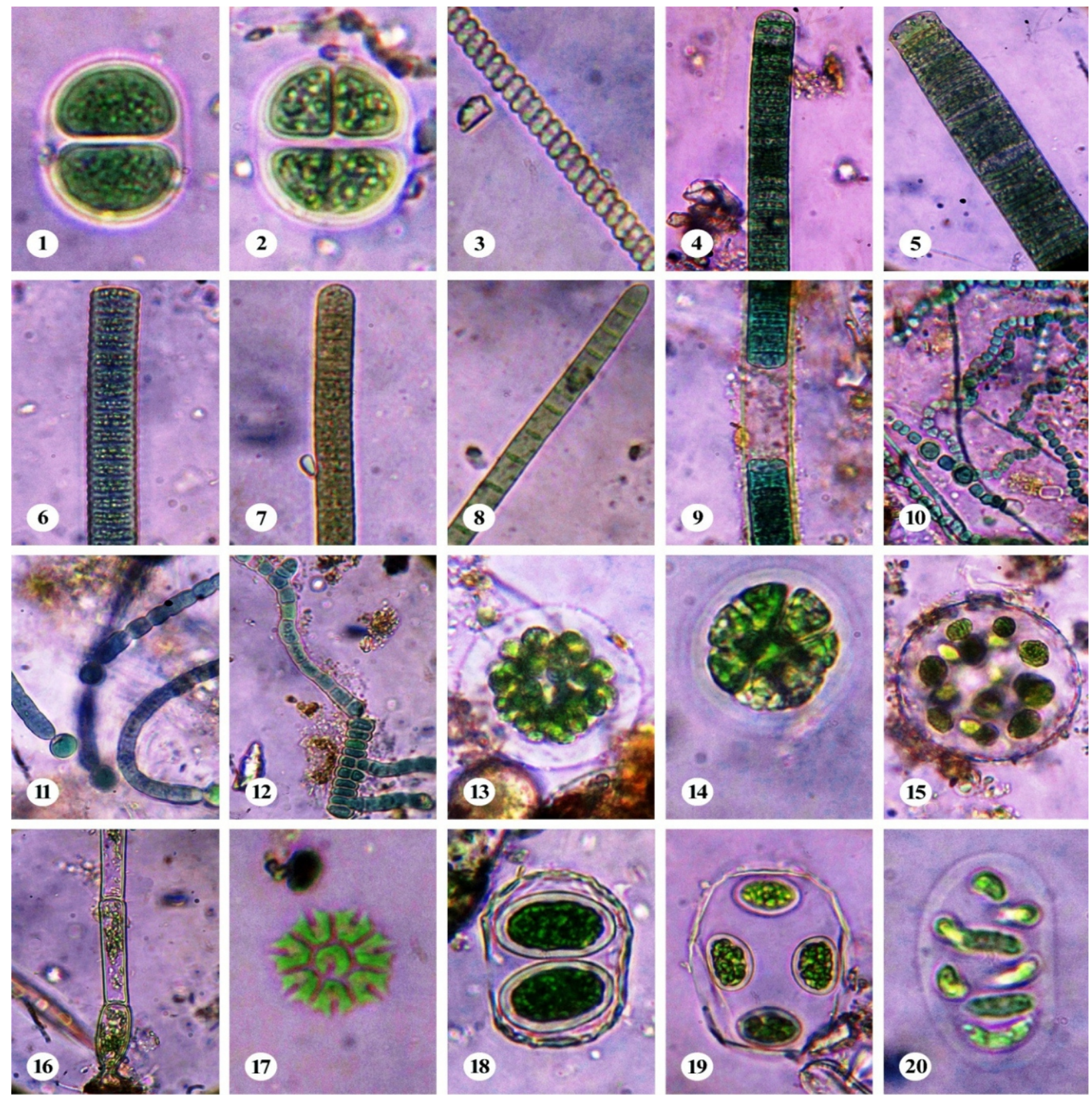

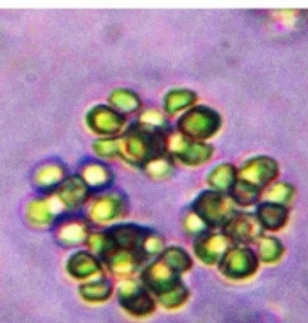

21
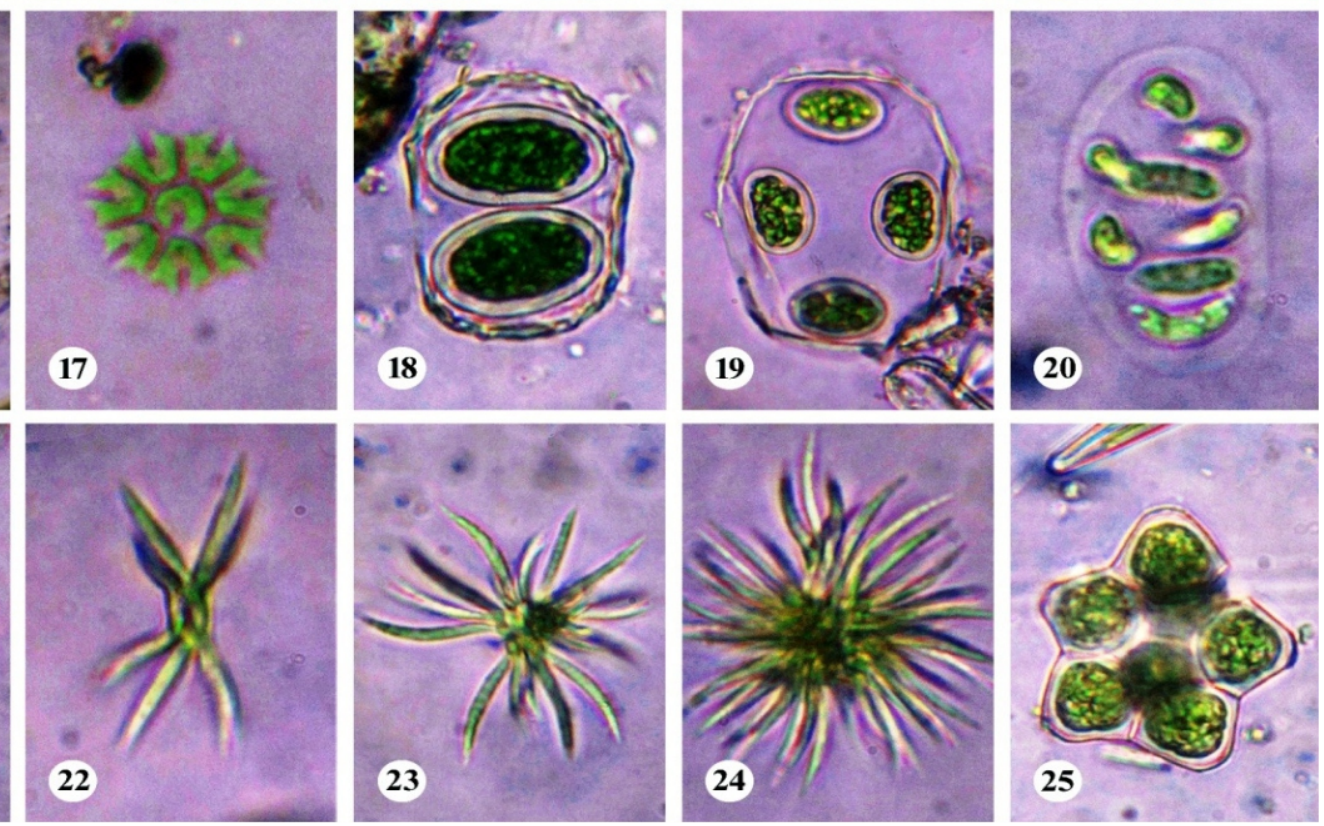

Figures: 1-2. Chroococcus turgidus 3. Spirulina subsalsa 4. Oscillatoria limosa 5. O. princeps 6. O. sancta 7. O. subbrevis 8. Phormidium autumnale 9. Lyngbya birgei 10. Nostoc microscopicum 11. Gloeotrichia echinulata 12. Stigonema ocellatum 13. Eudorina elegans 14. Pandorina morum 15. Sphaerocystis schroeteri 16. Oedogonium sp. 17. Pediastrum tetras v. tetraodon 18-19. Gloeotaenium loitlesbergerianum 20. Nephrocytium agardhianum 21. Westella botryoides 22. Ankistrodesmus spiralis 223-24. A. spiralis v. fasciculatus 25. Coelastrum cambricum 

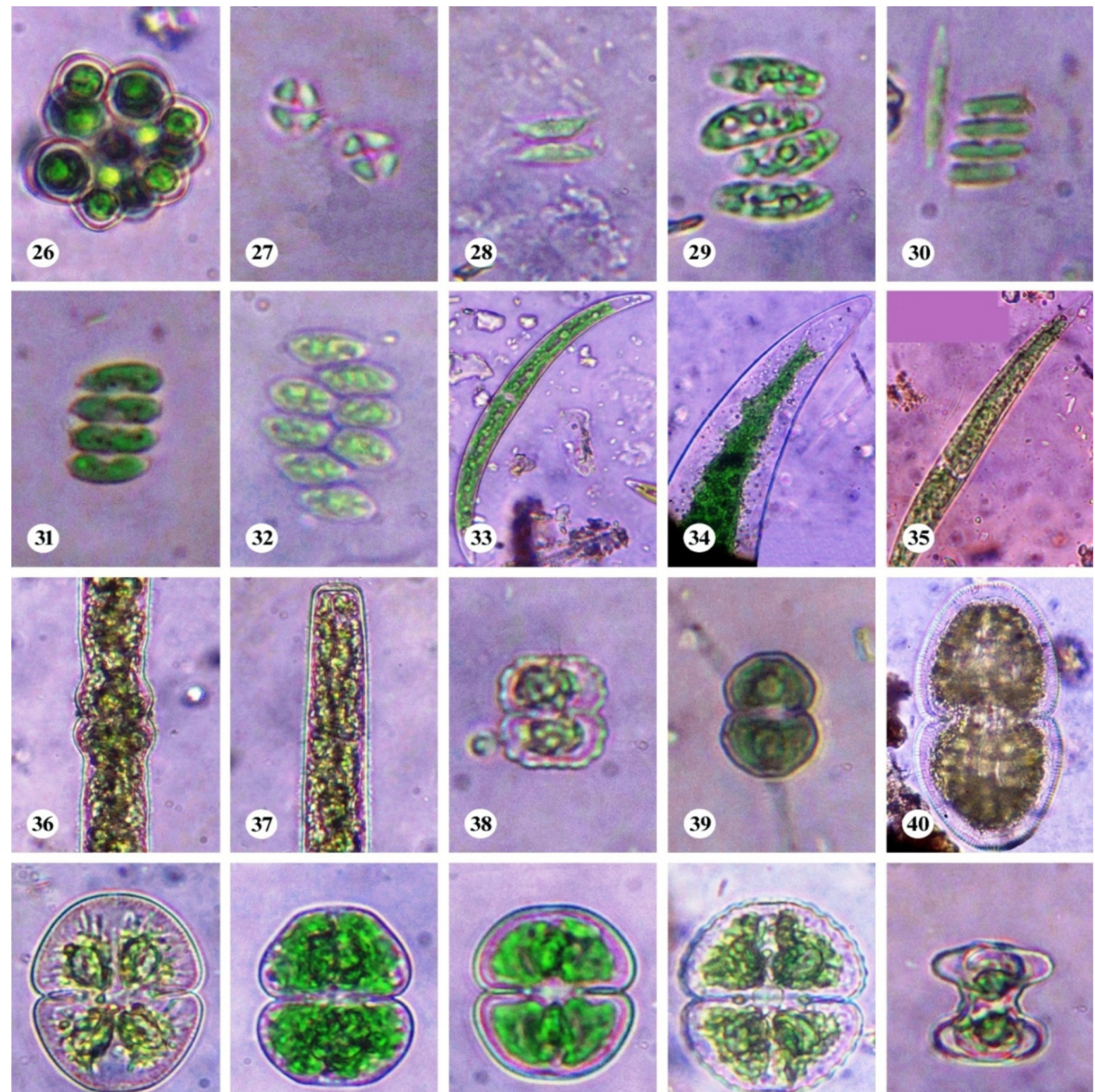

41
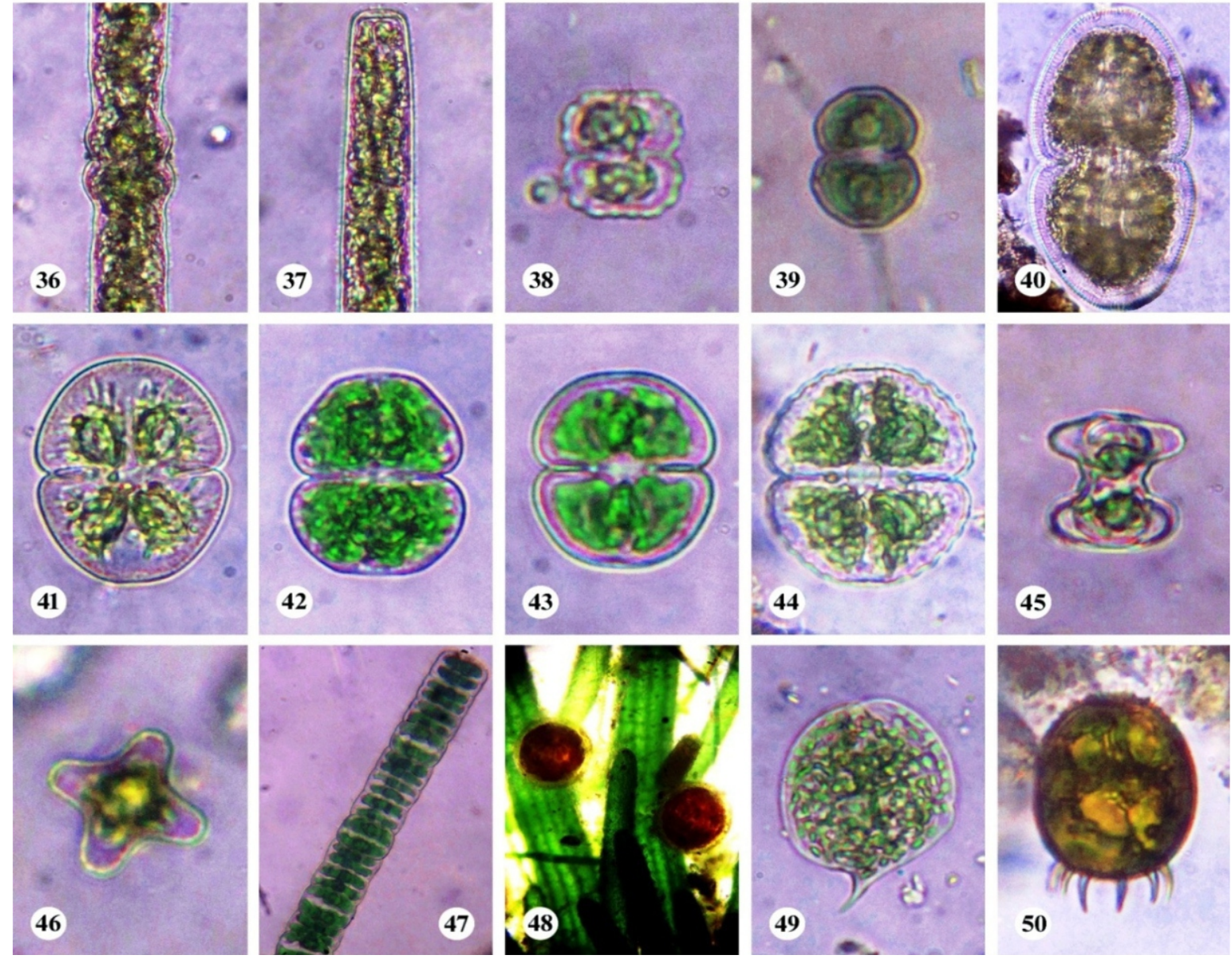

Figures: 26.Coelastrum cambricum v. intermedium 27. Crucigenia tetrapedia 28. Scenedesmus acutiformis 29. S. arcuatus 30. S. armatus v. boglariensis 31. S. bijugatus 32. S. bijugatus v. gravenitzii 33. Closterium dianae 34. C. ehrenbergii 35. C. praelongum v. brevius 36-37. Pleurotaenium trabecula 38. Cosmarium blyttii 39. C. impressulum 40. C. javanicum 41. C. lundellii v. circulare 42-43. C. lundellii v. ellipticum 44. C. sublateriundatum 45-46. Staurastrum disputatum v. sinense 47. Hyalotheca dissiliens 48. Chara sp. 49. Phacus pseudoswirenkoi 50. Trachelomonas armata v. longispina. 
Locality no: 5, Sample no: 10, Date of collection: 2072/09/05.

Hyalotheca dissiliens (Smith) ex Bréb. in Ralfs (Fig. 47) Tiffany and Britton 1952, P. 204, P1. 56, fig. 631

Filaments straight, cylindrical with wide sheath; cells cylindric-discoidal, 10-39 $\mu \mathrm{m}$ long, 10-33 $\mu \mathrm{m}$ broad, faintly constricted, lateral margins slightly convex.

Locality no: 4, Sample no: 08, Date of collection: 2072/09/05

\section{Chara sp.(Fig. 48) Prescott 1951, P. 336.}

Stem and branchlets corticated; branchlets 4 segmented; bract cells 4 or more at a node; antheridium below the oogonium, both from periphery of branchlet nodes.

Locality no: 4, Sample no: 08, Date of collection: 2072/09/05.
Phacus pseudoswirenkoi Prescott (Fig. 49) Prescott 1951, P. 402, P1. 85, Fig. 26; P1. 87, Fig. 2; P1. 88, Fig. 14.

Cells orbicular, $41 \mu \mathrm{m}$ long, $30 \mu \mathrm{m}$ broad; anterior broadly rounded, posterior caudus short, sharp and curved; periplast longitudinally striated; paramylon single, large circular disc.

Locality no: 4, Sample no: 08, Date of collection: 2072/09/05.

Trachelomonas armata var. longispina (Playf.) Def. (Fig. 50) Prescott 1951, P. 411, P1. 83, Fig. 27.

Test broadly ovate, $40-43 \mu \mathrm{m}$ long, $30 \mu \mathrm{m}$ broad; without collar but with a circle of erect spines at margin; anterior spines short but posterior spines stout and both short and long.

Locality no: 5, Sample no: 05, Date of collection: 2072/04/12.

\section{Conclusions}

The study showed that the algal flora of Hasina wetland area was rich. Out of 44 algae reported from this wetland, desmids were dominant with five genera. As usual, genus Cosmarium was dominant with six species. The rare but interesting algae reported here were Spirulina subsalsa,
Nostoc microscopicum, Stigonema ocellatum, Crucigenia tetrapedia and Staurastrum disputatum. Further extensive exploration is essential to identify more important algae of this area that may help for further documentation and conservation.

\section{Acknowledgments}

Authors are thankful to the Chairman, Department of Botany, Post Graduate Campus, Tribhuvan University, Biratnagar for laboratory facilities. We also acknowledge the people of wetland area for their kind cooperation and help during algae collection.

\section{References}

Bando T, Nakano T, Watanabe M. The desmid flora of Kathmandu, Nepal. Bull Natn Sci Mus, Ser B, Tokyo, $1989,15,1-25$.

Bharati SG and Hegde GR. Desmids from Karnataka State and Goa, Part III. Genus Cosmarium Corda. Nova Hedwigia, 1982, 36, 733-57.

Croasdale H and Flint EA. Flora of New Zealand, Freshwater algae, Chlorophyta, Desmids with ecological comments on their habitats, Vol. I. Wellington, Govt Print, New Zealand., 1986.

Croasdale H and Flint EA. Flora of New Zealand, Freshwater algae, Chlorophyta, Desmids with ecological comments on their habitats, Vol. II. Botany Division, D.S.I.R., Christchurch, New Zealand, 1988.

Croasdale H, Flint EA, Racine MM. Flora of New Zealand, Freshwater algae, Chlorophyta, Desmids with ecological comments on their habitats, Vol. III. Manaaki Whenua Press, Lincoln, Canterbury, New Zealand, 1994.

Desikachary TV. Cyanophyta, monograph on algae, ICAR,
New Delhi, 1959.

Guiry MD and Guiry GM. AlgaeBase. World-wide electronic publication, National University of Ireland, Galway. http://www.algaebase.org; 2018, accessed in January 2018

Hirano M. Fresh water algae from East Nepal. Study reported of Baika Junior College, 1984, 32, 197-215.

Hirano M. Fresh water algae from Langtang Himal, Nepal Himalaya. Contr. Biol. Lab., Kyoto Univ., Japan, 1969, 22, 1-42.

Hirano M. Fresh water algae from the Nepal Himalaya, collected by a member of the Japanese Climbing Expedition. Contr. Biol. Lab., Kyoto Univ., Japan, 1963, $16,1-23$

Jha S and Kargupta AN. Taxonomy of genus Oscillatoria Vaucher from the river Koshi basin. In: Environment and plant: Glimpses of research in South Asia. Jha, P.K. et al. (eds.), 2006, 264-74.

Juttner I, Sharma S, Dahal BM, Ormerod SJ, Chimonides PJ, 
Cox EJ. Diatoms as indicators of stream quality in the Kathmandu valley and Middle hills of Nepal and India, Freshwater Biology, 2003, 48, 2065-2084.

Komarek J. Contribution to the Chlorococcal algae of Cuba. Nova Hedwigia 1983, 37(1), 65-180.

Kouwets FAC. Desmids from the Auvergne (France). Hydrobiol., 1987, 146, 193-263.

Necchi O Jr, West JA, Rai SK, Ganesan EK, Rossignolo NL, Goër SL de. Phylogeny and morphology of the freshwater red alga Nemalionopsis shawii (Rhodophyta, Thoreales) from Nepal. Phycological Research, Japan, 2016, 64, 1118. Doi: $10.1111 /$ pre. 12116

Nurul Islam AKM and Yusuf Haroon AK. Desmids of Bangladesh. Int. Revue ges. Hydrobiol., 1980, 65(4), 551604.

Philipose MT. Chlorococcales, monograph on algae, ICAR, New Delhi, 1967.

Prasad BN and Misra PK. Fresh water algal flora of Andaman and Nicobar islands, 1992, Vol. II, B. singh and M.P. Singh Publ., Dehradun, India.

Prescott GW. Algae of the western great lakes area. WM.C. Brown Publishers, Dubuque, Iowa, 1951.

Rai SK. Additional desmids (Chlorophyceae) to eastern flora of
Nepal. Nepalese J. of Biosciences, 2014, 4, 40-6.

Rai SK. Algal flora of Betana wetland, Morang, Nepal. Nepalese Journal of Biosciences, 2011a, 1, 104-13.

Rai SK. Algal flora of Koshi Tappu Wildlife Reserve including some new species to Nepal. Nepalese Journal of Integrated Sciences, 2013, 3, 26-32.

Rai SK. Spirulina: A green food and medicine for future world. Pragyik, 2011, 2, 174-183.

Scott AM and Prescott GW. Indonesian desmids. Hydrobiologia, 1961, 17(1-2), 1-132.

Sharma OP. Textbook of Algae. Tata Mc. Graw Hill Publishing Company Ltd., New Delhi, 1992.

Shrestha S, Rai SK, Dhakal MR. Algae of Itahari Municipality and its adjoining area, eastern Nepal. Int. Journal of Applied Sciences and Biotechnology, 2013, 1(1), 5-10.

Siwakoti M, Shrestha R, Basnet TB, Sharma LN. Wetlands Inventory of Chure Region (East of Narayani), 2012, 42-4.

Tiffany LH and Britton ME. The algae of Illinois. Hafner publishing Co., New York, 1952.

Van Den Hoek C, Mann DG and Jahns HM. Algae: An introduction to phycology. Cambridge University Press. First South Asian Edition, 2009. 